\title{
APROVECHAMIENTO DEL ESTIÉRCOL DE ALPACA E ICHU PARA LA PRODUCCIÓN DE BRIQUETAS COMO FUENTE DE ENERGÍA CALORÍFICA EN AREQUIPA
}

\author{
USE OF MANURE FROM ALPACA AND ICHU FOR THE PRODUCTION \\ OF BRIQUETTES AS A SOURCE OF HEAT ENERGY IN AREQUIPA
}

\author{
Juan Palo Tejada ${ }^{1}$, Alicia Puma Taco $^{1}$ (D), Enriqueta Campos Falcon ${ }^{1}$

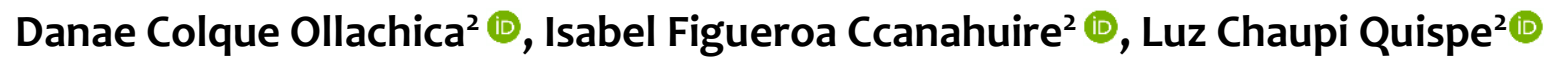

\author{
${ }^{1}$ Ciencias Naturales y Formales, Universidad Nacional San Agustín de Arequipa, Perú \\ ${ }^{2}$ Escuela Profesional de Ingeniería Ambiental, Universidad Nacional de San Agustín de Arequipa, Perú
}

Recibido (Received): 23 / $01 / 2021$ Aceptado (Accepted): 22/06 / 2021

\begin{abstract}
RESUMEN
En este trabajo se realizaron pruebas para determinar el poder calorífico de briquetas de estiércol de alpaca e ichu y aglomerante; la proporción de la mezcla está en $80 \%$ de estiércol de alpaca, 18\% de ichu, 2\% de cal y agua en medida proporcional al estiércol. Los resultados muestran la temperatura máxima que alcanza la briqueta en estudio es $500{ }^{\circ} \mathrm{C}$ medido con un termómetro infrarrojo y poder calorífico estimado para estas briquetas $4318,18 \mathrm{cal} / \mathrm{g}$. Por los resultados obtenidos las briquetas resultantes se pueden utilizar como combustible alternativo y económico y además amigable con el medio ambiente. El estiércol de alpaca a 4000 m.s.n.m. no se utiliza como abono para la agricultura en la población de San Antonio de Chuca-Imata.
\end{abstract}

Palabras Clave: Briquetas, Estiércol de alpaca, Ichu, Poder calorífico, Friabilidad.

\section{ABSTRACT}

In this work, tests were carried out to determine the calorific power of briquettes of alpaca and ichu manure and binder; the proportion of the mixture is $80 \%$ alpaca manure, $18 \%$ ichu, $2 \%$ lime and water in proportion to the manure. The results show the maximum temperature reached by the briquette under study is $500{ }^{\circ} \mathrm{C}$ measured with an infrared thermometer and estimated calorific power for these briquettes $4318.18 \mathrm{cal} / \mathrm{g}$. Due to the results obtained, the resulting briquettes can be used as an alternative and economical fuel that is also friendly to the environment. Alpaca manure at 4000 meters above sea level is not used as fertilizer for agriculture in the town of San Antonio de Chuca-Imata.

Keywords: Briquettes, Alpaca manure, Ichu, Calorific value, Friability.

\section{INTRODUCCIÓN}

El consumo desmedido de los recursos naturales por los seres humanos ha causado grandes estragos en el medio ambiente. Este trabajo se centra en el aprovechamiento del estiércol de alpaca; que se crían como ganado en las partes altas de Arequipa, como es en el distrito de San Antonio de Chuca - Imata; aquí se crían llamas, alpacas y ovejas; en esta localidad, el estiércol no se usa en la agricultura debido a la altura que está sobre los 4000 m.s.n.m. y la temperatura media en el año oscila entre $-10^{\circ} \mathrm{C}$ por la noche y en el día hasta $12{ }^{\circ} \mathrm{C}$; por lo que el estiércol del ganado no puede ser usado como abono para la agricultura.

1 Corresponding author.:

E-mail: jpalot@unsa.edu.pe
Nuestro interés es la elaboración de briquetas de estiércol, para ser usados en los hogares de esta localidad como combustible para la cocción de sus alimentos. Valderrama et al. [1] estudió el poder calorífico de briquetas de estiércol de cuy con aglomerados, la combustión de las briquetas alcanza temperaturas medias superficiales entre valores de $250^{\circ} \mathrm{C}-400{ }^{\circ} \mathrm{C}$; esta variación se debe a que la cal y la arcilla logran disminuir el poder calorífico de las briquetas y lo hacen gradualmente de acuerdo al porcentaje en peso en su composición; el punto de inflamación de las briquetas fluctúan entre $86-90{ }^{\circ} \mathrm{C}$ y poder calorífico de $10.7 \mathrm{MJ} / \mathrm{kg}$. Paniego [2] estudio la combustión del excremento de ganado, como combustible único en hogares rurales de España. Beraztegui et al. [3] concluye que los valores de durabilidad se logran usando carbón en porcentajes de 7 a $25 \%$, aglomerante menor al $6 \%$ y contenido de 
biomasa hasta un $70 \%$. Mendoza et al. [4] concluye que la elaboración de briquetas con un 100\% de estiércol de ganado bovino, no es viable debido al alto contenido de cenizas que este presenta. Valiente [5] los resultados para briquetas en base a residuos de arroz, con humedad de $48.53 \%$, contenido de ceniza de $9.89 \%$ y poder calorífico de $14.1 \mathrm{MJ} / \mathrm{kg}$. Suryaningsih et al. [6] concluye que para incrementar la combustión de las briquetas las partículas deben ser de dimensiones pequeñas (malla 100).

\section{MATERIALES Y METODOLOGÍA}

\subsection{El proceso de fabricación}

La biomasa utilizada para el estudio es estiércol de alpaca e ichu como material base para la fabricación de briquetas, los cuales fueron recolectados del distrito de San Antonio de Chuca - Imata ( $15^{\circ} 55^{\prime} 43^{\prime \prime} \mathrm{S} 71^{\circ} 06^{\prime} 31^{\prime \prime O}$ ), Perú. Los aditivos fueron agua y cal como aglomerante. El primer paso es el proceso de molido mecánico y refinado del tamaño de las partículas del estiércol utilizando el tamiz de malla $1 \mathrm{~mm}$, con el fin de garantizar la compactación y el acabado superficial para la mezcla. Para compactación de las briquetas se ejerció presión en moldes cilíndricos huecos con dimensiones de $8 \mathrm{~cm}$ de diámetro y $7.5 \mathrm{~cm}$ de alto y un radio interno de $2 \mathrm{~cm}$. Finalmente estas fueron desmoldadas y expuestas al sol en el Distrito de San Antonio de Chuca - Imata, durante un periodo de 6 días.

TABLA I

Porcentajes de composición para el estiércol de alpaca, ichu, cal y número de briquetas.

\begin{tabular}{cccc}
\hline & Briqueta & (Alc) & \\
\hline Componente & & Formulación & Cantidad \\
\hline Estiércol de Alpaca & 80 & 2000 & (unidades) \\
Ichu & 18 & 450 & 27 \\
Cal & 2 & 50 & \\
Subtotal & 100 & 2500 & \\
Cantidad de Agua & & 2100 \\
\hline
\end{tabular}

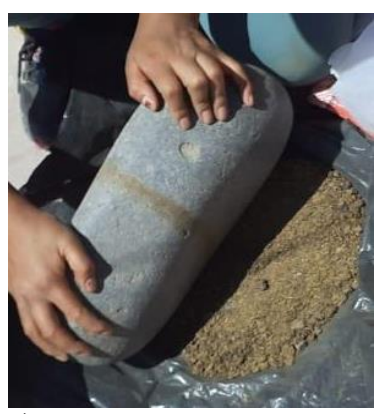

a)

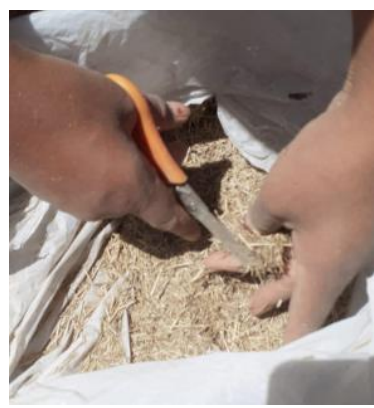

c)

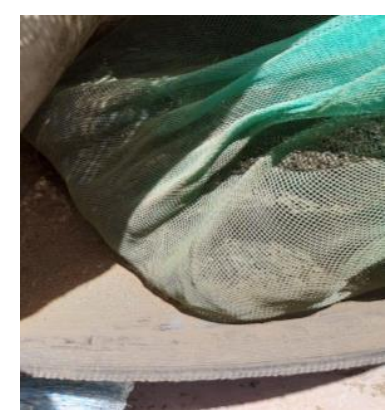

b)

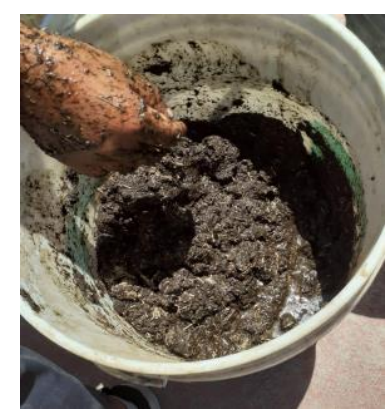

d)
Fig. 1. Muestra el procedimiento para la elaboración de las briquetas: a) Molienda mecánica del estiércol de alpaca, b) Tamizado del estiércol, c) ichu cortado y d) mezcla de estiércol, ichu triturado, cal y agua como material adhesivo.

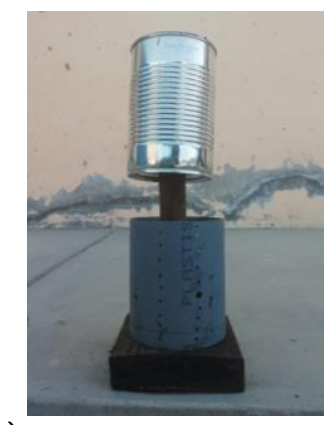

a)

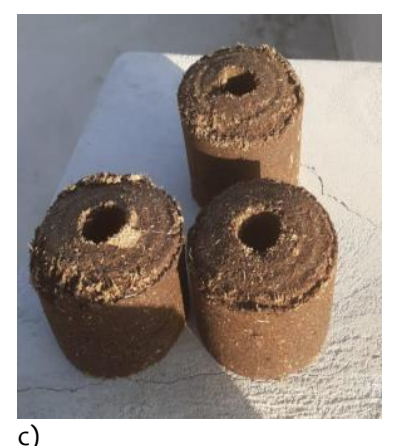

c)

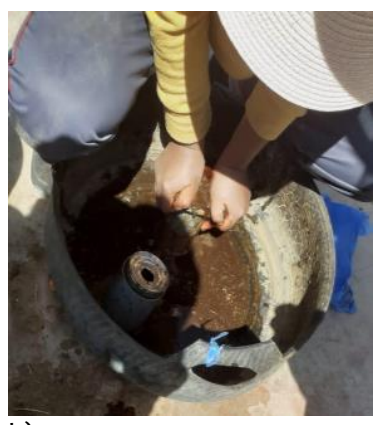

b)

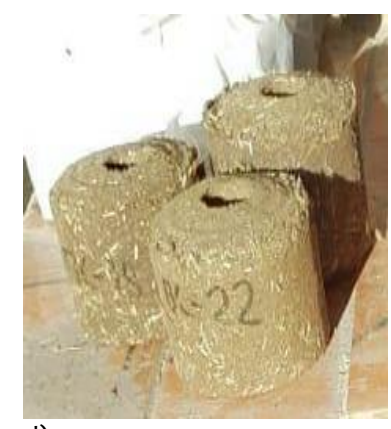

d)
Fig. 2. a) Molde b) Compactación de la muestra, c) secado, d) briquetas listas para usarse. Las briquetas fueron expuestas a temperatura ambiente, durante un periodo de 6 días.

\subsection{Contenido de humedad y densidad}

Para Castells [7], la humedad es el factor más crítico en los combustibles de biomasa, porque determina la energía que se puede obtener durante la combustión. Si el contenido de humedad es alto, el poder calorífico es menor. Para determinar el $\mathrm{CH}$ se 
midió la masa de la briqueta seca (PBs) y la masa de la briqueta húmeda (PBh) [8]. El contenido de humedad se calculó según la ecuación (1):

$$
C H(\%)=\frac{P B_{h}-P B_{S}}{P B_{S}}
$$

Donde $\mathrm{CH}$ es el contenido de la humedad, $P B_{s}$ es la masa de las briquetas secas en gramos, $P B_{h}$ es la masa de las briquetas húmedas en gramos.

Para determinar su densidad, se usa la ecuación (2):

$\rho=\frac{\text { Masadelabriqueta }}{\text { Volumendelabriqueta }}$

\subsection{Prueba térmica}

La prueba térmica es parte esencial para conocer la tasa de combustión de las briquetas. La temperatura de combustión de la muestra se mide de acuerdo a la ecuación (3) [6]:

$$
T C=\frac{M b}{\text { Tiempodecombustión }}
$$

Además, $M b \quad(g)=M_{i}(g)-M_{r}(g)$

Donde TC es la tasa de combustión, $M b$ es masa de briquetas en llamas, $M_{i}$ es la masa inicial de briquetas, $M_{r}$ es la masa de briquetas restante (ceniza).

\subsection{Evaluación del poder calorífico de la briqueta por calentamiento del agua}

Para estimar el poder calorífico, se quemó $30 \mathrm{~g}$ de briquetas en una cocina diseñada para este propósito, para transferir calor a la olla que contiene $1000 \mathrm{~g}$ de agua y registrar el aumento de temperatura que alcanza el agua. El calor se calculó según las ecuaciones (4) y (5).

$Q=m \cdot c \cdot\left(T_{2}-T_{1}\right)$

$P_{c}=\frac{Q}{M_{b}}$

\subsection{Contenido de Cenizas}

La ceniza es un componente que se obtiene a partir de la combustión de las briquetas de acuerdo a la ecuación (6) [9]:

$$
C C(\%)=100-\frac{M_{O}-M}{M_{\text {So }}} \times 100 \%
$$

Dónde: $C C=$ contenido de ceniza $M_{o}=$ masa de la muestra inicial y del platillo antes de incinerar (g); $M=$ masa del platillo y ceniza (g); $M_{s o}=$ masa de la muestra antes de la ceniza (g).

\section{6 Índice de Friabilidad}

Es el índice de resistencia al impacto (IRI), el cual consiste en dejar caer repetidas veces la briqueta desde una altura de $2 \mathrm{~m}$ o 1,8 $\mathrm{m}$. Es el cociente entre el promedio del número de caídas y el promedio del número de trozos formados, si hay rupturas, dando una idea bastante aproximada de la resistencia de las briquetas. Este ensayo se considera como el más útil para predecir la resistencia global de una briqueta [10], el cual fue calculado según la ecuación (7):

$$
I F R=\sum_{i=1}^{n} \frac{N L}{N P} x 100
$$

Dónde: NL es el número de lanzamientos, NP es el número de pedazos (briqueta fraccionada).

La norma utilizada como base para la realización de este ensayo es la ASTM D440 - 86. El mínimo valor para que las briquetas (biocombustible) sean aceptables comercialmente es IFR=50.

\section{RESULTADOS Y DISCUSIÓN}

\subsection{Resultados de la densidad y contenido de humedad}

Tabla II

Propiedades mecánicas y químicas de la briqueta de estiércol de alpaca e ichu

\begin{tabular}{cccc}
\hline Masa & Dimensiones & Densidad & CH \\
\hline$\times 10^{-3}(\mathrm{~kg})$ & $\mathrm{x} 10^{-3}(\mathrm{~m})$ & $\left(\mathrm{kg} / \mathrm{m}^{3}\right)$ & $(\%)$ \\
\hline \multicolumn{4}{c}{ Diámetro externo } \\
$75,70 \pm 0.02$ & & \\
$88.0 \pm 0.1$ & Diámetro interno & & \\
& $22.10 \pm 0.02$ & 298,4 & 10.9 \\
& Alto & \\
& $70.80 \pm 0.02$ & & \\
\hline
\end{tabular}

\subsection{Resultados de la Prueba Térmica}

Para la prueba térmica se utilizaron briquetas de $88 \mathrm{~g}$ y para medir la temperatura se utilizó un termómetro infrarrojo (FLUKE). Los resultados se muestran en la Fig.3.

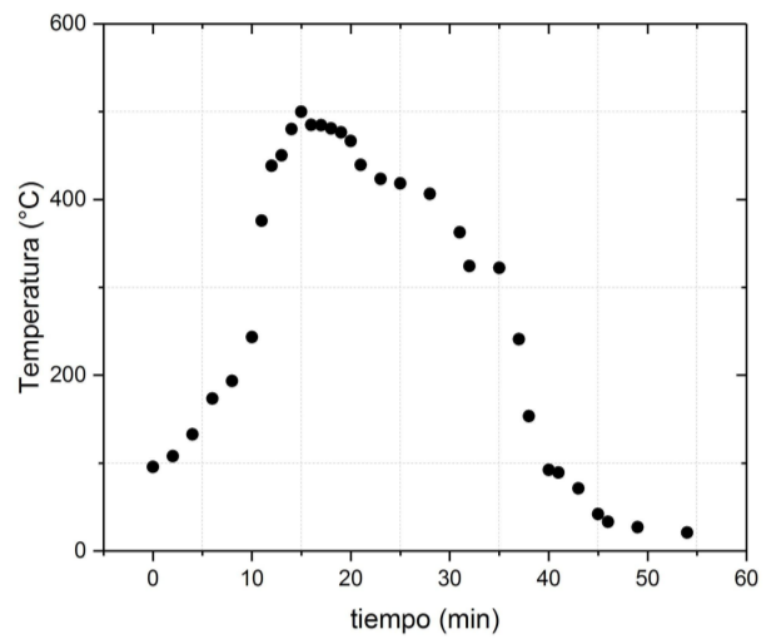

Fig. 3. Temperatura de combustión de la briqueta en función del tiempo. Se muestra la temperatura máxima que alcanza los $500^{\circ} \mathrm{C}$ a los 16 minutos. 
La tasa de combustión indica la velocidad a la cual se consume la briqueta en un segundo. Entonces según la ecuación (3) obtenemos:

$$
\begin{gathered}
\text { Tasadecombustión }(\text { briqueta })=\frac{(88-19) g}{3240 s} \\
\text { Tasadecombustión }(\text { briqueta })=0.0213 \frac{g}{s}
\end{gathered}
$$

\subsection{Prueba de la briqueta para calentar el agua}

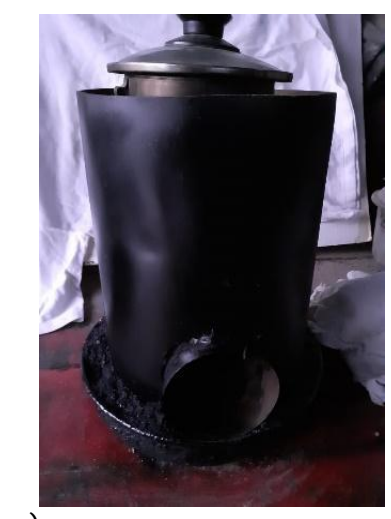

a)

Fig. 4. a) Muestra la disposición de la cocina, olla que contiene agua y termocupla para medir la temperatura. b) se observa la combustión completa de la briqueta.

La cantidad de agua en la olla fue de $1000 \mathrm{~g}$ y la masa de briquetas empleada para calentar el agua fue $30 \mathrm{~g}$ con un tiempo de 2100 segundos. La briqueta tuvo una combustión completa debido a la forma de la briqueta de estiércol de alpaca e ichu. Además, esta tiene un orificio en el medio, permitiendo una buena circulación de oxígeno.

Considerando el poder calorífico del eucalipto igual a 17.1 MJ/kg [11], con el fin de estimar el poder calorífico de la briqueta en estudio y empleando la tabla número III, la cual muestra la eficiencia de la cocina empleada.

TABLA III

Muestra la eficiencia de la cocina empleada, la masa de agua es 1000 g

\begin{tabular}{ccccc}
\hline$m_{L}$ & $P_{c}$ & $Q_{L}$ & $Q_{H 2 O}$ & $\eta$ \\
$(\mathrm{g})$ & $(\mathrm{MJ} / \mathrm{kg})$ & $(\mathrm{kJ})$ & $(\mathrm{kJ})$ & $(\%)$ \\
\hline 21 & 17.1 & 358.5 & 159.2 & 44.4 \\
\hline
\end{tabular}

Al haber estimado la eficiencia de la cocina podemos obtener el poder calorífico estimado para la briqueta de estiércol de alpaca e ichu. Considerando la masa de agua $1000 \mathrm{~g}$ y la masa de la briqueta $30 \mathrm{~g}$, empleamos la ecuación (5).

$$
Q_{H 2 O}=m . c .\left(T_{2}-T_{1}\right)
$$

$$
\begin{gathered}
Q_{H 2 O}=1000 \mathrm{~g} \frac{1 \mathrm{cal}}{g^{\circ} \mathrm{C}}(67-10)^{\circ} \mathrm{C}=57000 \mathrm{cal} \\
Q_{H 2 O}=238.73 \mathrm{KJ} \\
\eta=\frac{Q_{H 2 O}}{Q_{B r}} \\
Q_{B r}=\frac{57000}{0,44}=129545,45 \mathrm{cal}=542.59 \mathrm{KJ} \\
P_{B R}=\frac{Q_{B r}}{m_{B r}}=4318,18 \mathrm{cal} / \mathrm{g}=18.1 \mathrm{MJ} / \mathrm{kg}
\end{gathered}
$$

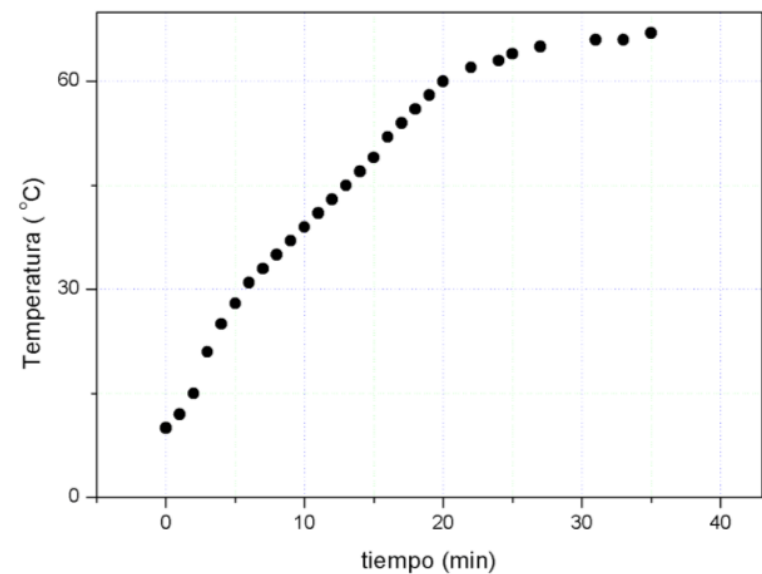

Fig. 5. Muestra la curva temperatura del agua en función al tiempo con las briquetas Alc.

\subsection{Resultados de la prueba de friabilidad}

Los resultados para obtener el índice de friabilidad son obtenidos utilizando la ecuación (7). Los resultados se muestran en la tabla IV.

TABLA IV

Registro y resultados del índice de friabilidad.

\begin{tabular}{ccc}
\hline $\begin{array}{c}\text { Número de } \\
\text { lanzamientos }\end{array}$ & $\begin{array}{c}\text { Número de } \\
\text { pedazos }\end{array}$ & IFR \\
\hline 5 & 3 & 166.7 \\
\hline
\end{tabular}

En la tabla IV se observa, que el índice de friabilidad es de 166,7 para las briquetas de estiércol de alpaca, comprobando que el IFR es mayor al mínimo aceptable resultando satisfactorio este ensayo y demostrando una buena resistencia al impacto.

\subsection{Costo y gastos para la fabricación de las briquetas}

TABLA V

Insumos utilizados en la fabricación de briquetas de estiércol de alpaca

\begin{tabular}{cccc}
\hline $\begin{array}{c}\text { Estiércol } \\
\text { de Alpaca }\end{array}$ & Ichu & Cal & $\begin{array}{c}\text { Número } \\
\text { total de } \\
\text { Briquetas }\end{array}$ \\
\hline $200 \mathrm{~g}$ & $45 \mathrm{~g}$ & $5 \mathrm{~g}$ & 3 \\
$1800 \mathrm{~g}$ & $405 \mathrm{~g}$ & $45 \mathrm{~g}$ & 27 \\
\hline
\end{tabular}


TABLA VI

Precio de insumos utilizados para la fabricación de briquetas de estiércol de alpaca.

Gasto de producción de las briquetas.

\begin{tabular}{cc}
$1,8 \mathrm{~kg}$ de E. de alpaca & $\mathrm{S} / .2,80$ \\
405 g de Ichu & $\mathrm{S} / .0,60$ \\
$45 \mathrm{~g}$ de cal & $\mathrm{S} / .0,40$ \\
3 moldes de briquetas & $\mathrm{S} / \cdot 7,00$ \\
\hline Total & $\mathrm{S} / .10,80$
\end{tabular}

\section{CONCLUSIONES}

- El poder calorífico estimado de estas briquetas de estiércol de alpaca e ichu es de $18.1 \mathrm{MJ} / \mathrm{kg}$. Se considera que el $44 \%$ de la energía contenida en la briqueta se transfiere al agua, el otro $56 \%$ se pierde al ambiente.

- La temperatura máxima alcanzada en la combustión de las briquetas es $500^{\circ} \mathrm{C}$.

- La combustión de las briquetas es uniforme y su llama es azulada lo que indica una combustión completa según las observaciones realizadas esto debido al orificio del diseño de las briquetas que permite la mejor circulación del oxígeno.

- El estiércol de los animales cuando no se puede utilizar como abono natural, como biomasa se puede reutilizar, en las zonas alto andinas de nuestro país como material de combustión para la cocción de alimentos.

- El costo calculado para 1,8 kg de material para la elaboración de las briquetas es 10.80 soles, en comparación con briquetas comerciales de hojarasca de $2 \mathrm{~kg}$ está en 15,50 soles y de carbón vegetal en 13 soles.
- Las briquetas son aptas para el transporte debido a que su índice de friabilidad es de 176.7, lo que indica una buena resistencia al impacto.

\section{AGRADECIMIENTOS}

Agradecemos a la Escuela Profesional de Física de la Universidad Nacional de San Agustín de Arequipa por brindarnos los equipos para realizar las mediciones correspondientes.

\section{REFERENCIAS}

[1] A. Valderrama et al., "Briquetas de Residuos Sólidos Organicos como Fuente de Energia Calorifica en cocinas no Convencionales", en XVII CONIMERA, Lima, 2007.

[2] P. Paniego, "Combustión de excrementos. Una aproximación experimental”, Revista Historia Autónoma, no. 8, pp. 13-25, 2016.

[3] C. Berastegui et al., "Elaboración de biocombustibles sólidos densificados a partir de tusa de maíz, bioaglomerante de yuca y carbón mineral del departamento de Córdoba", Rev. Chil. Ing., vol. 25, no. 4, pp. 643-653, 2017.

[4] J. M. Mendoza et al., "Fabricación de biocombustibles sólidos densificados (briquetas) a base de aserrín de acacia y estiercol de bovinos en el departamento de Córdoba”, Rev. Chil. Ing., vol. 28, no. 3, pp. 448-460, 2020.

[5] A. Valiente, "Elaboración de briquetas Para aprovechamiento del residuo de arroz en beneficios del Municipio de el progreso", Tesis de grado, Facultad de Ingeniería, URL, Guatemala, 2017.

[6] S. Suryaningsih et al., "The analysis of ignition and combustion properties of the burning briquettes made from mixed biomass of rice husk and corn cob", IOP Conf. Ser.: Mater. Sci. Eng., vol. 550, no. 1, 2019. [7] X. Castells, Tratamiento y Valoración energética de residuos. Madrid, España: Ed. Díaz de Santos, 2005.

[8] W. Samame, "Determinación del poder calorífico de briquetas de carbón utilizando cantidades de residuos de biomasa”, Tesis de grado, Facultad de Ingeniería, UCV, Chiclayo, 2017.

[9] L. Ifa et al., "Techno-economic analysis of bio-briquette from cashew nut shell waste", Heliyon, vol. 6, no. 9, 2020.

[10] V. Vicente, "Estudio de la influencia del porcentaje de arcilla en la calidad de las briquetas de hojas de caña de azúcar mediante ensayos físicos y térmicos", Tesis de grado, Facultad de Ciencias e Ingeniería, PUCP, Lima, 2017.

[11] L. Soria, "Evaluación de un sistema integrado cocina a leña con tanque de agua para calefacción de vivienda rurales en zonas alto andinas del Perú", Tesis de Maestría, Facultad de Ciencias Naturales y Formales, UNSA, Arequipa, 2019. 